\title{
Neoplasia intra-epitelial córneo-conjuntival (NIC) - Relato de um caso atípico
}

\author{
Conjunctival corneal intraepithelial neoplasia (CIN)-Report of an atypical case
}

\author{
Luciene Alves da Silva Santos ${ }^{1}$ \\ Renata Leal Barbosa ${ }^{2}$ \\ Luciene Barbosa de Sousa ${ }^{3}$
}

Trabalho realizado no Hospital Oftalmológico de Sorocaba - SP

${ }^{1}$ Médica assistente junto ao Setor de Córnea e Doenças Externas do Hospital Oftalmológico de Sorocaba.

${ }^{2}$ Médica residente do Hospital Oftalmológico de Sorocaba.

${ }^{3}$ Chefe do Serviço de Residência Médica do Hospital Oftalmológico de Sorocaba. Doutora em Oftalmologia pela Universidade Federal de São Paulo - UNIFESP. Chefe do Setor de Doenças Externas e Córnea do Departamento de Oftalmologia da Universidade Federal de São Paulo - UNIFESP.

Endereço para correspondência: Renata Leal Barbosa, Rua T55 Q 95 Lt 12 Setor Bueno - Goiânia (GO) CEP 74215170

E-mail: renatalealb@uol.com.br

Recebido para publicação em 21.02.2003

Versão revisada recebida em 12.04.2004

Aprovação em 23.04.2004

\section{RESUMO}

As autoras relatam um caso de NIC com apresentação e epidemiologia atípicos, bem como sua evolução com diferentes tratamentos. A paciente feminina, 41 anos, encaminhada ao departamento de córnea e doenças externas do HOS, queixava-se de baixa acuidade visual em olho direito (OD) e foi submetida a exame oftalmológico completo. Ao exame, detectou-se em ambos os olhos opacidades corneanas epiteliais digitiformes e, diante da hipótese de NIC, realizaram-se 3 ciclos de 14 dias de colírio de mitomicina C a $0,02 \%$ 4x/dia em OD e ressecção associada a crioterapia em olho esquerdo (OE). Observamos recidiva em OD, após a qual realizamos ressecção associada a crioterapia com nova recidiva, seguida de nova ressecção e crioterapia, sem recidiva até o último exame (seguimento de 3 anos). Não houve recidiva em OE.

Descritores: Neoplasias da conjuntiva/quimioterapia; Córnea/patologia; Mitomicina com uso terapêutico; Crioterapia

\section{INTRODUÇÃOO}

A neoplasia intra-epitelial córneo-conjuntival (NIC) é uma doença benigna do epitélio de superfície ocular, com baixo potencial de malignidade, de difícil diagnóstico (podendo apresentar-se sob as mais variadas formas) e de difícil tratamento ${ }^{(1)}$.

O termo NIC hoje substitui outros antes empregados, como doença de Bowen, epitelioma intra-epitelial, displasia, carcinoma in situ e intra-epitelioma ${ }^{(1)}$.

A NIC compreende duas entidades: a displasia escamosa, em que as células atípicas atingem somente parte do epitélio de superfície, e o carcinoma in situ, no qual as células atípicas atingem toda a espessura do epitélio, poupando a membrana basal. O tratamento de escolha para a NIC compreende excisão e crioterapia. Recentemente, vários estudos têm mostrado que o uso de quimioterápicos como a mitomicina $\mathrm{C}$, interferon e 5-fluorouracil podem eliminar NICs de variados tamanhos ${ }^{(2-4)}$.

Se houver invasão sub-epitelial da córnea ou conjuntiva (células anaplásicas ultrapassando a membrana basal), a lesão passa a ser chamada de carcinoma invasivo de células escamosas ${ }^{(2)}$.

Nós aqui relatamos um caso atípico de NIC, com acometimento bilateral em que foram realizadas diferentes modalidades de tratamento para cada olho.

\section{RELATO DE CASO}

Paciente L.T., 41 anos, branca, natural de Tietê-SP, procedente de Porto Feliz-SP, profissão do lar, apresentou-se ao ambulatório de Córnea e Doen- 


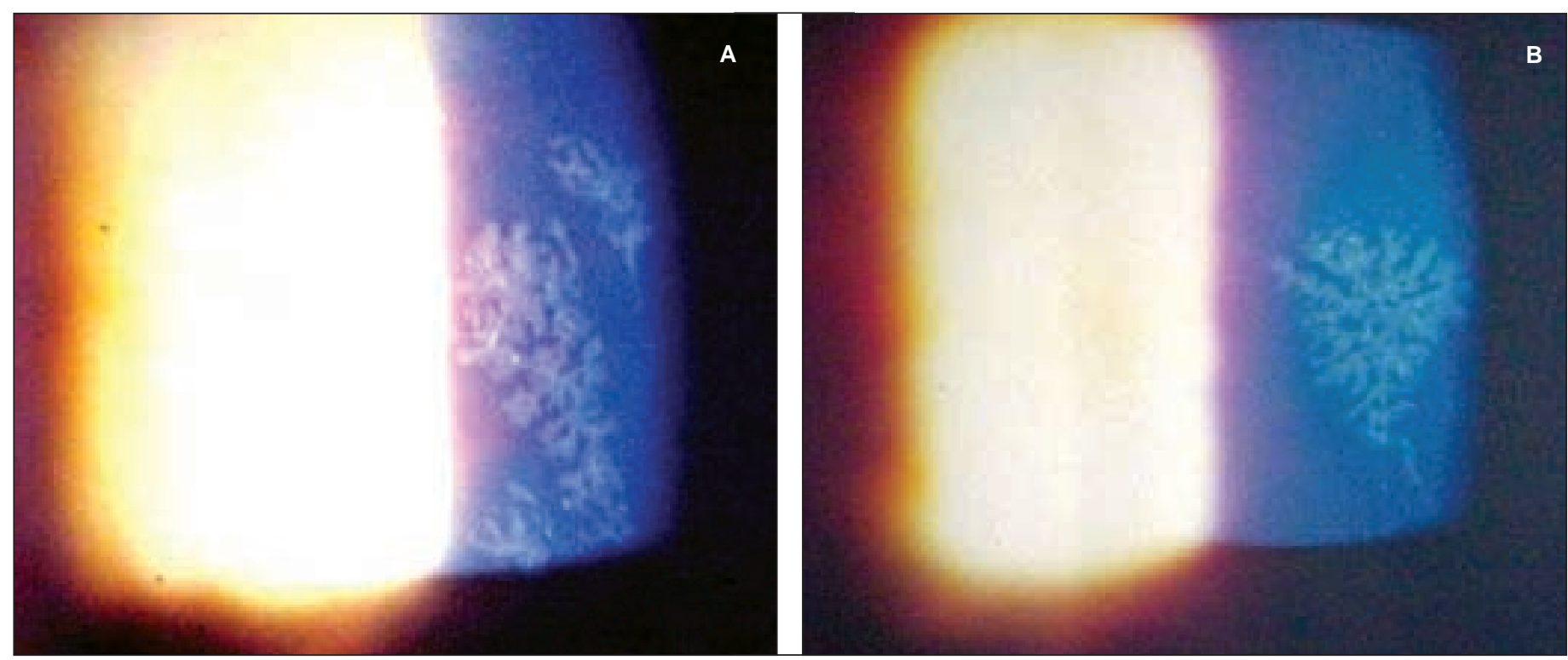

Figura 1 - A e B - Lesões com aspecto arboriforme, dispersas na córnea

ças Externas do Hospital Oftalmológico de Sorocaba em março de 2000, com queixa de prurido e baixa acuidade visual em olho direito de início há um ano.

Antecedentes pessoais/familiares/oftalmológicos, nada digno de nota.

Ao exame oftalmológico apresentava acuidade visual (com correção) de 0,3 em olho direito (OD) e de 1,0 em olho esquerdo (OE). À biomicroscopia apresentava em OD espessamento límbico, microvilosidades sub-epiteliais e opacidade epitelial das 10 às $11 \mathrm{~h}$ atingindo o eixo visual e opacidade epitelial das 4 às $5 \mathrm{~h}$ e, em OE, pinguécula nasal, área de leucoplasia perilímbica nasal medindo aproximadamente $0,2 \times 0,2 \mathrm{~mm}$ e opacidade intra-epitelial nasal inferior.

Demais exames oculares apresentavam-se sem alterações.

Foi feita hipótese diagnóstica de neoplasia intra-epitelial córneo-conjuntival (NIC) em ambos os olhos.

Em OD iniciou-se o tratamento com mitomicina C $0,02 \%$, quatro vezes ao dia por quatorze dias, sendo três ciclos com intervalo de dez dias entre o primeiro e o segundo e de dois meses entre o segundo e o terceiro. Após dois meses do término do $2^{\circ}$ ciclo a paciente já atingia acuidade visual corrigida igual a 1,0. Observou-se, no início de cada ciclo, ceratite puntata moderada e hiperemia conjuntival leve. Após dois meses do fim do terceiro ciclo houve recidiva, tendo sido indicada excisão com margem de segurança e crioterapia das 7 às $11 \mathrm{~h}$ a $2 \mathrm{~mm}$ do limbo, com exame anátomo-patológico mostrando processo inflamatório crônico inespecífico. Três meses após a cirurgia observou-se a segunda recidiva em OD, na qual as lesões apresentavam aspecto arboriforme, dispersas na córnea (Figura 1 - A e B). Indicou-se, então, nova excisão com crioterapia e novo estudo anátomo-patológico que mostrou displasia moderada. Até a última visita, com 3 anos de seguimento, não havia sinais de nova recidiva.
Em OE foi realizada excisão e crioterapia em região córneolimbar nasal com exame anátomo-patológico mostrando displasia moderada. Não houve recidiva até a última visita (julho de 2003).

\section{DISCUSSÃO}

Vários são os fatores de risco associados à NIC. Dentre eles, os mais importantes são a pele clara (como a de nossa paciente) e a exposição à radiação ultra-violeta. A presença do HPV sorotipos 16 e 18, a exposição ao petróleo e ao tabaco, o uso de lentes de contato e a infecção pelo HIV também têm sido implicados em sua patogênese $\mathrm{e}^{(1-2,4)}$.

Os homens idosos são os mais acometidos, principalmente durante a sétima década de vida ${ }^{(1-2)}$. A paciente em questão foge ao padrão epidemiológico mais encontrado por ser do sexo feminino e ter, à época do diagnóstico, 41 anos.

A doença é definida como sendo de apresentação uni ou bilateral, sendo esta mais rara ${ }^{(5)}$. Neste caso os dois olhos estavam acometidos pelo tumor simultaneamente.

A transformação maligna ocorre em 5\% dos casos, evoluindo para carcinoma espino-celular. Este é o tumor maligno conjuntival mais freqüentemente encontrado em nosso meio ${ }^{(6)}$.

Displasia epitelial corneana primária é uma condição rara que afeta principalmente o epitélio da córnea, sem a presença de uma massa limbar. Provavelmente surge no limbo e, por razões desconhecidas, tem maior chance de expandir-se sobre a córnea do que de acumular-se no limbo ou estender-se para a conjuntiva. O epitélio anormal parece opaco ou acinzentado, geralmente avascular e tem bordas lisas ou digitiformes. As lesões podem ser múltiplas, estendendo-se do limbo, ou dispersas na córnea com aspecto arboriforme ${ }^{(7)}$. No caso descrito observamos este padrão na segunda recidiva do olho direito. 
A displasia da conjuntiva pode se apresentar como um espessamento gelatinoso devido à acantose. Na modalidade descrita como leucoplasia, apresenta lesão sob forma de placa branca na mucosa causada por hiperqueratose e às vezes com aspecto papilomatoso.

O diagnóstico é eminentemente clínico, feito através do encontro de lesões córneo-conjuntivais típicas, que coram com rosa bengala e pode ser confirmado através de estudo anátomo-patológico ${ }^{(2,8-9)}$.

Se estas lesões forem negligenciadas por longos períodos, aumentam as chances de ocorrer um envolvimento extenso da córnea $^{(7,9)}$.

O tratamento tradicionalmente realizado para a doença corneana tem sido a excisão associada à crioterapia. A excisão pode levar à cicatrização córneo-conjuntival, formação de membranas retro-corneais e semeadura de células neoplásicas, podendo também resultar em excisão parcial. Isoladamente, leva à recorrência em um terço dos casos, num intervalo médio de dois $\operatorname{anos}^{(5,10)}$. A crioterapia pode levar a cicatrização, membrana retro-corneal, disfunção endotelial e edema, devendo ser reservada somente para as lesões peri-límbicas ${ }^{(5)}$. Associada à ressecção, leva à recorrência em $9 \%$ dos pacientes. O fator mais importante na prevenção da recorrência é a presença de margens cirúrgicas livres e não o tipo histológico de $\mathrm{NIC}^{(1)}$.

Devido às complicações destas modalidades de tratamento, surgiram modalidades alternativas, tais como a mitomicina $\mathrm{C}$ tópica, a radiação beta, o interferon alfa- $2 \mathrm{~b}$ perilesional $\mathrm{e}$ tópico ${ }^{(3-5)}$.

A mitomicina $\mathrm{C}$ é um alquilante potente, altamente tóxico, que inibe a síntese de DNA, erradicando a NIC. Pode ser usada como primeira opção de tratamento ou para tratar recidivas ou lesões parcialmente tratadas. Os efeitos colaterais mais observados são: hiperemia leve, alergia ocular, lacrimejamento, fotofobia, epiteliopatia puntata e dor. Em cobaias, foi comprovado efeito carcinogênico, o que deve ser informado ao paciente previamente ao tratamento ${ }^{(6,10)}$.

$\mathrm{Na}$ dose de $0,02 \%$ a mitomicina $\mathrm{C}$ erradica a maioria das lesões e tem baixa toxicidade se usada por até 14 dias. Após isso, ocasiona dor, blefaroespasmo, hiperemia conjuntival, simbléfaro, erosões corneanas e uveíte. Portanto, são preconizados ciclos de no máximo 14 dias de administração tópica da droga, com intervalo de, no mínimo dez dias, podendo ser prescritos no máximo três ciclos ${ }^{(10)}$.

Realizamos no olho esquerdo da paciente excisão e crioterapia, após o que não houve recidiva (seguimento de três anos) ou complicações pós-operatórias, havendo confirmação histopatológica do diagnóstico.

No olho direito, onde optamos por mitomicina C tópica a $0,02 \%$, após 2 meses do fim do terceiro ciclo, houve recidiva da lesão e observamos como efeitos colaterais durante o início de cada ciclo, ceratite puntata e hiperemia conjuntival leve. Foi realizada excisão com crioterapia e o estudo histo-patológico mostrou processo inflamatório crônico inespecífico. Após, foi observada recidiva da lesão do OD, realizada nova excisão e crioterapia com erradicação da lesão e sem recidivas até o momento (três anos de seguimento).
A época de aparecimento das recidivas pode variar de um mês até onze anos, o que nos faz concluir que o seguimento dos pacientes portadores de NIC deve ser feito até o fim da $\operatorname{vida}^{(1-2)}$.

Com base no caso descrito, ressaltamos a importância do exame detalhado do olho contralateral, ainda que a doença do primeiro olho seja de rara apresentação bilateral e a queixa seja monocular. Reafirmamos a importância do diagnóstico clínico, mesmo frente a um primeiro resultado histo-patológico que descarte a hipótese aventada e exemplificamos a já descrita dificuldade de cura deste tumor de baixa virulência.

\section{ABSTRACT}

The authors report a case of NIC with atypical presentation and epidemiology as well as its evolution with different modalities of treatment. A forty-one-year-old female was referred to the Department of Cornea and External Diseases complaining about low visual acuity in the right eye and was submitted to complete ophthalmologic examination. The patient presented with corneal epithelial opacities with pseudopodia-like extensions in both eyes. The hypothesis for the case was CCIN and the patient received 3 cycles of topical $0.02 \%$ mitomycin $\mathrm{C}$ drops 4 times daily for 14 days in the right eye and surgical excision associated with cryotherapy in the left eye. After detection of recurrence of the lesion in the right eye, this lesion was submitted to excision and cryotherapy, which was followed by recurrence. The patient underwent excision and cryotherapy in the right eye once more and there was no recurrence since then. The lesion in the left eye never recurred (3 years of follow-up).

Keywords: Conjunctival neoplasms/drug therapy; Cornea/ pathology; Mitomycin C/therapeutic use; Cryotherapy

\section{REFERÊNCIAS}

1. Tabin G, Levin S, Snibson G, Loughnan M, Taylor H. Late recurrences and the necessity for long-term follow-up in corneal and conjunctival intraepithelial neoplasia. Ophthalmology. 1997;104(3):485-92.

2. Slamovits T. Basic and Clinical Science Course: Section 8, 1996-1997. San Francisco: American Academy of Ophthalmology, 1996. p.275-8.

3. Rozenman Y, Frucht-Pery J. Treatment of conjunctival intraepithelial neoplasia with topical drops of mitomycin C. Cornea. 2000;19(1):1-6.

4. Vann RR, Karp CL. Perilesional and topical interferon alfa- $2 b$ for conjunctival and corneal neoplasia. Ophthalmology. 1999;106(1):91-7.

5. Jones DB, Wilhelmus KR, Font RL. Beta radiation of recurrent corneal intraepithelial neoplasia. Trans Am Ophthalmol Soc. 1991;89:285-301.

6. Shiratori CA, Rodrigues LD, Schellini AS, Marques MEA, Padovani CR. Tumores conjuntivais malignos - ocorrência na Região de Botucatu. Rev Bras Oftalmol. 2001;60(1):71-6.

7. Waring GO 3rd, Roth AM, Ekins MB. Clinical and pathologic description of 17 cases of corneal Intraepithelial neoplasia. Am J Ophthalmol. 1984;97(5):547-59.

8. Wilson FM 2nd. Rose bengal staining of epibulbar squamous neoplasms. Ophthalmic Surg. 1976;7(2):21-3.

9. Brown HH, Glasgow BJ, Holland GN, Foos RY. Keratinizing corneal intraepithelial neoplasia. Córnea. 1989;8(3):220-4.

10. Frucht-Pery J, Sugar J, Baum J, Sutphin JE, Pe'er J, Savir H et al. Mitomycin $\mathrm{C}$ treatment for conjunctival corneal intraepithelial neoplasia: a multicenter experience. Ophthalmology. 1997;104(12):2085-93. 